\title{
MicroRNA-1185 Induces Endothelial Cell Apoptosis by Targeting UVRAG and KRIT1
}

\author{
Haoyuan Denga Xia Chua Zhenfeng Song ${ }^{a}$ Xinrui Denga Huan Xua Yaxin Yea \\ Songtao Lia Qiao Zhang ${ }^{a}$ Changhao Sun ${ }^{a, b}$ Ying Lia,b \\ aDepartment of Nutrition and Food Hygiene, Public Health College, Harbin Medical University, Harbin, \\ ${ }^{b}$ Research Institute of Food, Nutrition and Health, Sino-Russian Medical Research Center, Harbin \\ Medical University, Harbin, China
}

\section{Key Words}

MiR-1185 • Apoptosis • Endothelium • UVRAG • KRIT1

\begin{abstract}
Background/Aims: Atherosclerosis is a multifactorial chronic disease and is the main cause of death and impairment in the world. Endothelial injury and apoptosis play a crucial role in the onset and development of atherosclerosis. MicroRNAs (miRNAs) have been proven to be involved in the pathogenesis of atherosclerosis. However, studies of the functional role of apoptosis-related miRNAs in the endothelium during atherogenesis are limited. Methods: Cell injury and apoptosis were measured in five types of cells transfected with miR-1185 or cotransfected with miR-1185 and its inhibitor. Bioinformatics analysis and a luciferase reporter assay were used to confirm the targets of miR-1185. The effects of the targets of miR-1185 on endothelial apoptosis were determined using small-interfering RNA. Results: In this study, we first report that miR-1185 significantly promoted apoptosis in endothelial cells but not in vascular smooth muscle cells and macrophages. A mechanistic analysis showed that ultraviolet irradiation resistance-associated gene (UVRAG) and krev1 interaction trapped gene 1 (KRIT1), targets of miR-1185, mediated miR-1185-induced endothelial cell apoptosis. Conclusion: The results revealed the impact of miR-1185 on endothelial apoptosis, suggesting that miR-1185 may be a potential target for the prevention and treatment of atherosclerosis.
\end{abstract}

(C) 2017 The Author(s)

Published by S. Karger AG, Basel

\section{Introduction}

Atherosclerosis, a chronic multi-factorial vascular disease, has become a primary cause of death worldwide [1]. Atherosclerosis lesions, which primarily occur in large and medium-sized arteries, can be present throughout life and cause stroke or infarction due to ischaemia in the brain, heart or extremities [2], and accumulating evidence has shown that injury and apoptosis in the vascular endothelium are critical events in the development

H. Deng and X. Chu contributed equally to this work.

Ying Li

KARGER
Department of Nutrition and Food Hygiene, Public Health College, Harbin Medical University, 157 Baojian Road, Nangang District, Harbin, Hei Longjiang province, 150081 (P. R. China); E-Mail liying_helen@163.com 
and pathogenesis of atherosclerosis [3, 4]. In the initial step of atherosclerosis, endothelial injury and apoptosis lead to lipid accumulation and inflammatory reactions that contribute to the formation of atherosclerotic lesions [5]. Although several studies have demonstrated that multiple cardiovascular risk factors induced endothelial injury and apoptosis [6-8], the molecular mechanisms of endothelial injury and apoptosis remain incompletely understood.

MicroRNAs (miRNAs) are small, endogenous RNA molecules $\sim 22$ nucleotides in length that anneal to imperfect complementary sequences in the 3'- untranslated region (3'-UTR) of their target mRNAs to mediate post-transcriptional gene expression [9]. miRNAs have been found to play a significant role in vascular biology and disease [10-12]. In particular, endothelial miRNAs have been suggested as potential drug targets or novel biomarkers in the prevention and treatment of vascular diseases, such as atherosclerosis, restenosis and thrombosis $[13,14]$. Santulli et al. reported that endothelial-specific miRNA modulates vascular integrity and provided the potential by using miRNA-based strategy as a novel therapeutic approach for the treatment of atherosclerosis [15]. Some studies have also revealed that miRNAs are involved in the injury and apoptosis of endothelial cells [16-19], but the precise roles and exact mechanisms of miRNAs are unclear and need to be elucidated.

In this study, miRNA microarray analysis showed that miR-1185 expression was dramatically increased in primary human umbilical vein endothelia cells (pHUVEC) treated with stearic acid, which is generally considered as an inducer of endothelial cell apoptosis [20]. A previous study suggested that miR-1185 is abundantly expressed in plasma, and miR1185 was predicted to regulate potential target genes involved in modulating transcription factors and the cell cycle [21]. Thus, we hypothesized that miR-1185 may be related to endothelial apoptosis. To identify the effects of miR-1185 on vascular injury, five types of cells, pHUVEC, human EA.hy926 endothelial cells, human aortic endothelial cells (HAEC), human umbilical vein smooth cells (HUVSMC) and human THP-1 myelomonocytic cells, were used in this study to first show that miR-1185 induced endothelial cell apoptosis. The study was also designed to elucidate the mechanisms underlying miR-1185-promoted endothelial apoptosis by focusing on the potential role of miR-1185 in the down-regulation of ultraviolet irradiation resistance-associated gene (UVRAG) and krev1 interaction trapped gene 1 (KRIT1/CCM1) expression.

\section{Materials and Methods}

\section{miRNAs profiling}

Total RNA was extracted from primary human umbilical vein endothelia cells (pHUVEC) treated with or without stearic acid $(200 \mu \mathrm{M})$ for $24 \mathrm{~h}$. The miRNAs microarray analysis was performed by KangChen Bio-tech (Shanghai, China). In brief, after RNA quantity measurement using the NanoDrop 1000 (Thermo Fisher Scientific Inc., Waltham, MA, USA), the samples were labeled using the miRCURYTM Hy3TM/Hy5TM Power labeling kit (Exiqon, Vedbaek, Denmark) and hybridized on the miRCURYTM LNA Array (v.18.0, Exiqon) according to manufacturer's instructions. Arrays were scanned using the Axon GenePix 4000B microarray scanner (Axon Instruments, Foster City, CA, USA). GenePix Pro 6.0 software (Axon) was used to obtain raw data and analyze array images. Replicated miRNAs were averaged and miRNAs that intensities $\geq 30$ in all samples were chosen for calculating normalization factor. Expressed data were normalized using the Median normalization. After normalization, the statistical significance of differentially expressed miRNA was analyzed by $t$-test. Different expression of miRNAs between pHUVEC cells treated without stearic acid (control group) and treated with stearic acid (SA group) were identified through fold change, which was calculated as the ratio between two group means using data before column-wise normalization was applied, with a threshold set for up- and down-regulated genes of a fold change $\geq 2.0$ with $P$ value $<0.05$. Finally, hierarchical clustering was performed to show distinguishable miRNA expression profiling among samples.

Cell culture, fatty acid treatments and transfection

pHUVEC were purchased from Allcells (Shanghai, China) and maintained in complete medium (Allcells). The human endothelial cells line EA.hy926 cells (ATCC, Manassas, VA, USA) were maintained 


\section{Cellular Physiology Cell Physiol Biochem 2017;41:2171-2182 \begin{tabular}{l|l} 
and Biochemistry Published 10.159/000475571 & $\begin{array}{l}\text { C) } 2017 \text { The Author(s). Published by S. Karger AG, Basel } \\
\text { www.karger.com/cpb }\end{array}$ \\
\hline
\end{tabular} \\ Deng et al.: MiR-1185 Induces Endothelial Apoptosis}

in Dulbecco's Modified Eagle's Medium (DMEM, Invitrogen, Carlsbad, USA) containing 10\% FBS (PAA Laboratories, Pasching, Austria). HAEC were purchased from BNCC (Beijing, China) and maintained in complete medium (Allcells). HUVSMC were obtained from Sciencell (Carlsbad, CA, USA) and incubated in basal medium supplemented with smooth muscle cell growth supplement (Sciencell) and 10\% FBS (PAA Laboratories). The human myelomonocytic cell line THP-1 was obtained from ATCC and cultured in RPMI 1640 (Invitrogen) containing 10\% FBS (PAA Laboratories). All cells were grown in an atmosphere of 5\% $\mathrm{CO}_{2}$ and $95 \%$ air at $37^{\circ} \mathrm{C}$.

Stearic acid and Palmitic acid (Sigma, St Louis, MO, USA) were prepared as previously described [22]. In brief, stearic acid or palmitic acid (Sigma, St Louis, MO) complex with BSA (3 mmol/l fatty acid: 1.5 $\mathrm{mmol} / \mathrm{l} \mathrm{BSA}$ ) was dissolved in ethanol and saponified with sodium hydroxide. After the sodium salt was dried, the sodium salt was re-suspended in saline and heated $80^{\circ} \mathrm{C}$ until it completely dissolved. When the solution was warm, $20 \%\left(\mathrm{w} / \mathrm{v}\right.$ ) BSA was added and the mixture was stirred at $50^{\circ} \mathrm{C}$ for $4 \mathrm{~h}$. Then, the complex was sterilized by filtering for further usage.

pHUVEC were treated with $0,50,100,150$ and $200 \mu \mathrm{M}$ stearic acid, and treated with $200 \mu \mathrm{M}$ palmitic acid for $24 \mathrm{~h}$. Meanwhile, HUVSMC and THP-1 cells were also exposed to $200 \mu \mathrm{M}$ stearic acid for $24 \mathrm{~h}$. After fatty acid treatment, the related indices were measured.

miR-1185 mimic and inhibitor were synthesized by RiboBio Co. (Guangzhou, China). The cells (pHUVEC, EA.hy926, HAEC, HUVSMC and THP-1) were transfected with $50 \mathrm{nM}$ of miR-1185 mimic or co-transfected with $50 \mathrm{nM}$ of miR-1185 and $100 \mathrm{nM}$ of miR-1185 inhibitor using Lipofectamine 2000 (Invitrogen) according to the manufacturer's instructions. Small-interfering RNAs (siRNA) against UVRAG or KRIT1 (UVRAG-siRNA and KRIT1-siRNA) were purchased from Santa Cruz Biotechnology (Santa Cruz, CA), and pHUVEC were transfected with UVRAG-siRNA or KRIT1-siRNA. The scrambled sequence was used as a negative control (NC). Forty-eight hours after transfection, the cells were used for the following experiments.

\section{Cell viability measurement}

Lactate dehydrogenase (LDH) release was used to measure cellular injury, and propidium iodide (PI) and Hoechst staining were performed to assess cell apoptosis, as described in our earlier study [22]. To measure LDH release, an LDH assay kit (Thermo Fisher Scientific Inc.) was used to examine culture media. For PI staining, the cells were stained in PI staining solution for $15 \mathrm{~min}$, and the resultant fluorescence was detected with flow cytometry (LSRFortessa, BD, Franklin Lakes, NJ, USA). For Hoechst staining, cells were fixed in $4 \%$ paraformaldehyde and then stained with $5 \mu \mathrm{g} / \mathrm{ml}$ Hoechst 33342 dye (Beyotime Biotechnology, Jiangsu, China) for $15 \mathrm{~min}$. Apoptotic cells were observed with a fluorescence microscope (Nikon, Tokyo, Japan).

\section{RNA isolation and quantitative PCR}

Total RNAs with miRNAs was isolated with the miRNeasy Mini Kit (Qiagen, Hilden, Germany) according to the manufacturer's protocol. The miScript SYBR Green PCR Kit (Qiagen) was used to perform qPCR, and the expression level of U6 was used as an internal control. Additionally, all primers were purchased from Qiagen.

\section{Western blot}

Western blot was performed as previously described [22] using the following primary antibodies: Caspase-3 and UVRAG (Cell Signaling Technology, Danvers, MA, USA) as well as KRIT1 and $\beta$-actin (Santa Cruz Biotechnology, Dallas, TX, USA). Anti-goat or anti-rabbit alkaline phosphatase-conjugated antibody (Promega, Madison, WI, USA) was used as a secondary antibody.

\section{Target prediction and Luciferase activity assay}

miR-1185 target genes were predicted with TargetScan 6.2 (http://www.targetscan.org/). The pmiRRB-REPORT ${ }^{\mathrm{TM}}$ dual luciferase reporter vectors carrying the wild-type (WT) or mutated (MUT) 3'-UTR of miR1185 target genes (UVRAG and KRIT1) were constructed (Ribio Co., Guangzhou, China). Human embryonic kidney 293 (HEK-293) cells (ATCC, Manassas, VA, USA) were co-transfected with 200 ng of recombinant plasmid, $50 \mathrm{nM}$ of miR-1185 mimic and $100 \mathrm{nM}$ of miR-1185 inhibitor. After $24 \mathrm{~h}$ of transfection, the luciferase activities were measured with a dual luciferase reporter assay kit (Promega, Madison, WI, USA) on a luminometer (GloMaxTM 20/20, Promega).

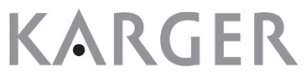




\section{Cellular Physiology Cell Physiol Biochem 2017;41:2171-2182 \begin{tabular}{l|l} 
and Biochemistry & $\begin{array}{l}\text { DOI: 10.1159/000475571 } 2017 \text { The Author(s). Published by S. Karger AG, Basel } \\
\text { www.karger.com/cpb }\end{array}$
\end{tabular} \\ Deng et al.: MiR-1185 Induces Endothelial Apoptosis}

\section{Statistical analysis}

Values expressed as the mean \pm SD were obtained from three separate experiments. The significance of differences was determined using a two-tailed Student's $t$ test or one-way ANOVA followed by the Student-Newman-Keuls (SNK) test, as appropriate. The SPSS 10.0 software (SPSS, Chicago, IL) was used for all statistical analyses. $P<0.05$ was considered to indicate a significant difference.

\section{Results}

Overexpression of miR-1185 induced damage and apoptosis in pHUVEC, EA.hy926 cells and HAEC

Our miRNA microarray data identified 48 up-regulated and 5 down-regulated miRNAs in the stearic acid-treated group compared with the control group (for all online suppl. material, see www.karger.com/doi/ 10.1159/000475571, Table S1 and Fig. S1). Among these miRNAs, miR-1185 expression increased the most, 8.78-fold in the stearic acid-treated group. Furthermore, qPCR verified that stearic acid dose-dependently up-regulated miR1185 expression in pHUVEC (see supplementary material, Fig. S2a). However, stearic acid did not up-regulate miR-1185 in HUVSMC and THP-1 cells; conversely, stearic acid reduced its expression (see supplementary material, Fig. S2b,c). These results suggest that stearic acid-mediated miR-1185 up-regulation may be specific to the cell type. Additionally, palmitic acid, an important saturated fatty acid, was also used to treat pHUVEC. Similar results were observed; palmitic acid $(200 \mu \mathrm{M})$ significantly increased miR-1185 expression in pHUVEC (see supplementary material, Fig. S2d). Stearic acid and palmitic acid are generally considered as inducers of endothelial apoptosis $[23,24]$; thus, we hypothesized that miR1185 may play an important role in endothelial injury and apoptosis.

pHUVEC, EA.hy926 endothelial cells and HAEC were used this study to examine the effect of miR-1185 on endothelial cells. As expected, the transfection of exogenous miR-1185 significantly increased miR-1185 expression in pHUVEC, and co-transfection with miR-1185

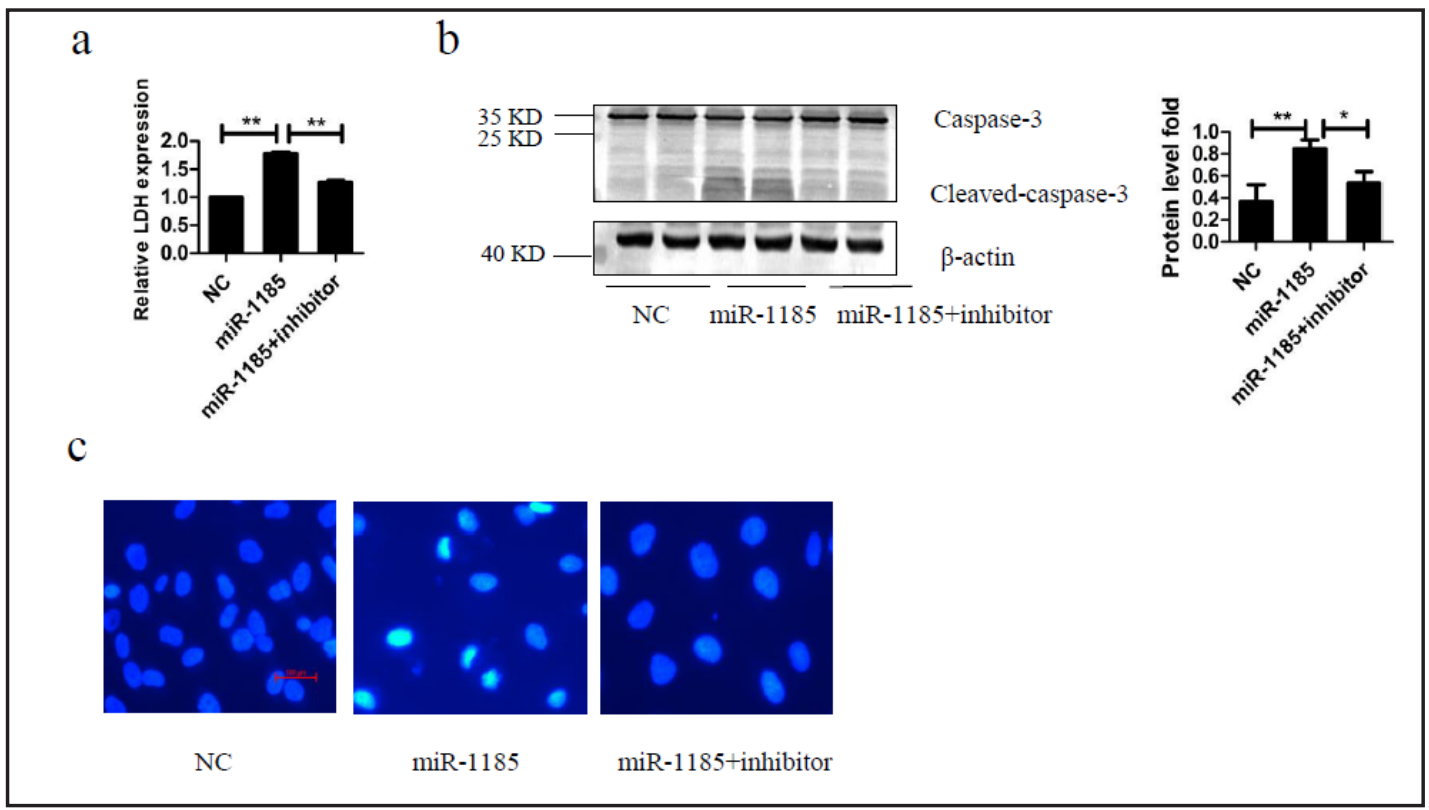

Fig. 1. The pro-apoptosis effect of miR-1185 on pHUVEC. The changes in LDH release (a), cleaved caspase-3

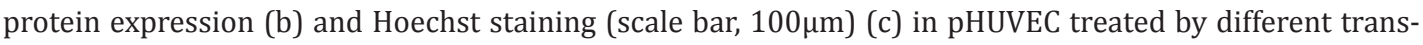
fection. pHUVEC were transfected with miR-1185 mimic or co-transfected with miR-1185 and its inhibitor. The scrambled sequence was used as a negative control (NC). After $48 \mathrm{~h}$ of transfection, LDH release, protein expression of cleaved caspase- 3 and Hoechst staining were detected. Each test was performed three times. ${ }^{*} P<0.05$ and ${ }^{* *} P<0.01$ for the indicated comparison. 
a

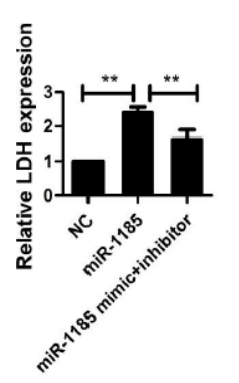

$\mathrm{c}$

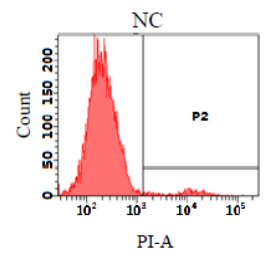

$\mathrm{b}$
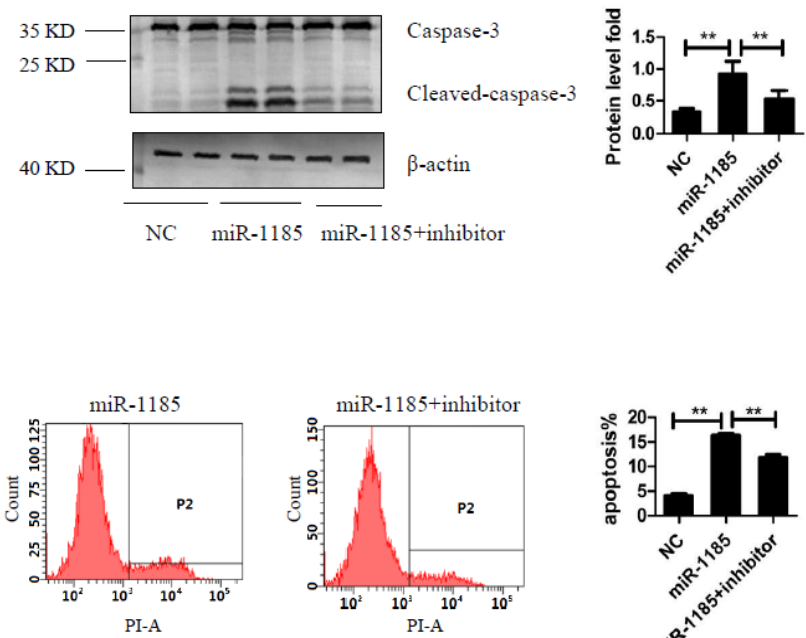

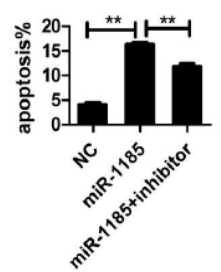

Fig. 2. The pro-apoptosis effect of miR-1185 on EA.hy926 cells. The changes in LDH release (a), cleaved caspase-3 protein expression (b) and flow cytometry with PI staining (c) in EA.hy926 cells treated by different transfection. EA.hy926 cells were transfected with miR-1185 mimic or co-transfected with miR-1185 and its inhibitor. The scrambled sequence was used as a negative control (NC). After $48 \mathrm{~h}$ of transfection, LDH release, protein expression of cleaved caspase-3 and PI staining were valuated. Each test was performed three times. ${ }^{* *} P<0.01$ for the indicated comparison.

inhibitor significantly decreased miR-1185 expression, suggesting successful transfection (see supplementary material, Fig. S3a). The transfection of miR-1185 significantly enhanced LDH release compared with the NC group; however, co-application with miR-1185 inhibitor almost entirely abrogated the effects of miR-1185 (Fig. 1a). Moreover, cell apoptosis was assessed based on the protein expression of cleaved caspase- 3 and Hoechst 33342 staining. Cleaved caspase-3 protein expression was elevated, and apoptotic morphological features, such as nuclear shrinkage and condensed chromatin, were evident in pHUVEC transfected with miR-1185, but these phenomena were reversed by co-transfection with miR-1185 inhibitor (Fig. 1b and c). Next, miR-1185 expression was measured in endothelial EA.hy926 cells by qPCR after different transfections, and the results were similar to those obtained in pHUVEC (see supplementary material, Fig. S3b). LDH release, cleaved caspase-3 expression and PI staining, as assessed by flow cytometry, were used to estimate cell injury and apoptosis. LDH release and cleaved caspase-3 expression were similar in EA.hy926 cells transfected with miR-1185 and co-transfection of its inhibitor (Fig. 2a and b). Moreover, the percentage of EA.hy926 cells undergoing apoptosis, as measured by PI staining, was dramatically increased in the miR-1185-transfected group, and co-transfection with miR-1185 inhibitor significantly abrogated the effect of miR-1185 on apoptosis (Fig. 2c). Furthermore, miR1185 expression was dramatically increased in HAEC transfected with miR-1185, and this change was attenuated in cells co-transfected with miR-1185 inhibitor (see supplementary material, Fig. S3c). LDH release and cleaved caspase-3 protein expression were increased in HAEC cells transfected with miR-1185, and these changes were attenuated by co-transfection with miR-1185 inhibitor (Fig. 3a and b). The results suggest that miR-1185 overexpression can induce endothelial cell damage and apoptosis.

Up-regulation of miR-1185 did not cause cellular injury and apoptosis in HUVSMC and THP-1 cells

The apoptosis of vascular smooth muscle cells and macrophages is well known to play crucial roles in the pathogenesis of atherosclerosis. The above results reveal that miR-1185 
a

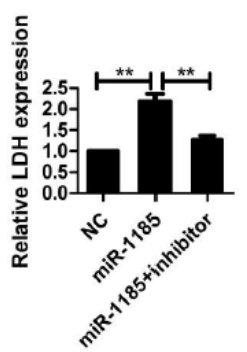

$\mathrm{b}$

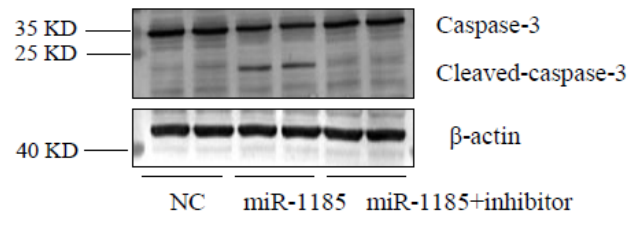

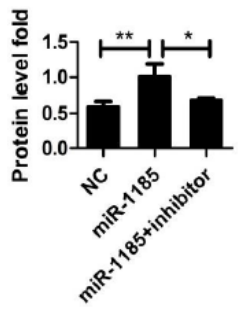

Fig. 3. The pro-apoptosis effect of miR-1185 on HAEC. The changes in LDH release (a), cleaved caspase-3 protein expression (b) in HAEC treated by different transfection. HAEC were transfected with miR-1185 mimic or co-transfected with miR-1185 and its inhibitor. The scrambled sequence was used as a negative control (NC). After $48 \mathrm{~h}$ of transfection, LDH release, protein expression of cleaved caspase- 3 were measured. Each test was performed three times. ${ }^{*} P<0.05$ and ${ }^{* *} P<0.01$ for the indicated comparison.

a

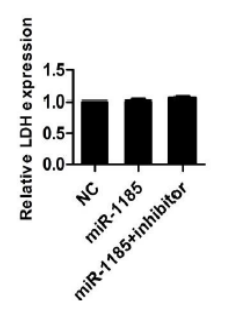

$\mathrm{b}$

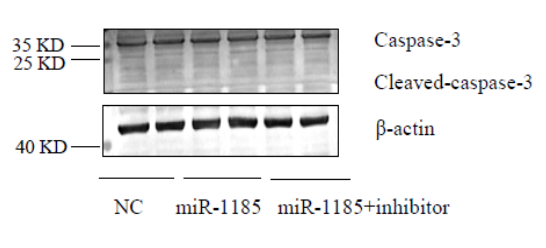

c

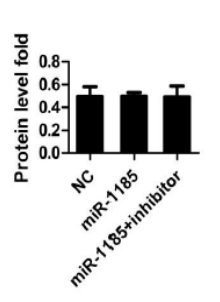

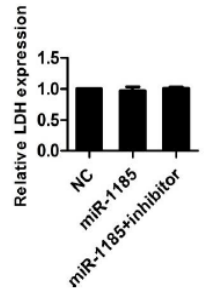

Fig. 4. The effect of miR-1185 on HUVSMC and THP-1 macrophages. The changes in LDH release (a) and cleaved caspase-3 expression (b) in HUVSMC treated by different transfection. The changes in LDH release in THP-1 macrophages (c) treated by different transfection. HUVSMC and THP-1 macrophages were transfected with miR-1185 mimic or co-transfected with miR-1185 mimic and its inhibitor. The scrambled sequence was used as a negative control (NC). After $48 \mathrm{~h}$ of transfection, LDH release and cleaved caspase-3 expression were measured. Each test was performed three times.

leads to endothelial cell apoptosis, but the effect of miR-1185 up-regulation on cell viability in vascular smooth muscle cells and macrophages was not known. To assess the relevance of the present findings to vascular smooth muscle cells and macrophages, the effect of miR1185 on HUVSMC and THP-1 cells was analysed. miR-1185 expression was significantly increased in HUVSMC and THP-1 cells transfected with miR-1185, but co-transfection with miR-1185 inhibitor down-regulated miR-1185 expression (see supplementary material, Fig. S3d,e). As shown in Fig. 4, HUVSMC transfected with miR-1185 did not exhibit significant injury or apoptosis compared with the NC group, as assessed based on LDH release (Fig. 4a) and cleaved caspase- 3 expression (Fig. 4b). THP-1 cells transfected with miR-1185 exhibited no change in LDH release (Fig. 4c). These results indicate that the up-regulation of miR-1185 does not significantly affect the viability of vascular smooth muscle cells and macrophages.

miR-1185 specifically suppresses the expression levels of UVRAG and KRIT1

Because miR-1185 promoted apoptosis in endothelial cells, we investigated the mechanism underlying this effect. To this end, a bioinformatics analysis was performed using an online algorithm, TargetScan 6.2, to predict the potential mRNA targets of miR-1185. Among the miR-1185 targets found to be associated with apoptosis, UVRAG and KRIT1 were of interest. UVRAG has been identified as a Bcl-2-associated X protein (BAX) suppressor that regulates apoptosis, and UVRAG deletion increases BAX-induced apoptosis in tumour cells [25]. Moreover, a loss of KRIT1 promotes apoptosis in the vascular endothelium of zebrafish embryos and in human endothelial cells [26]. TargetScan predicted that the binding sequence 


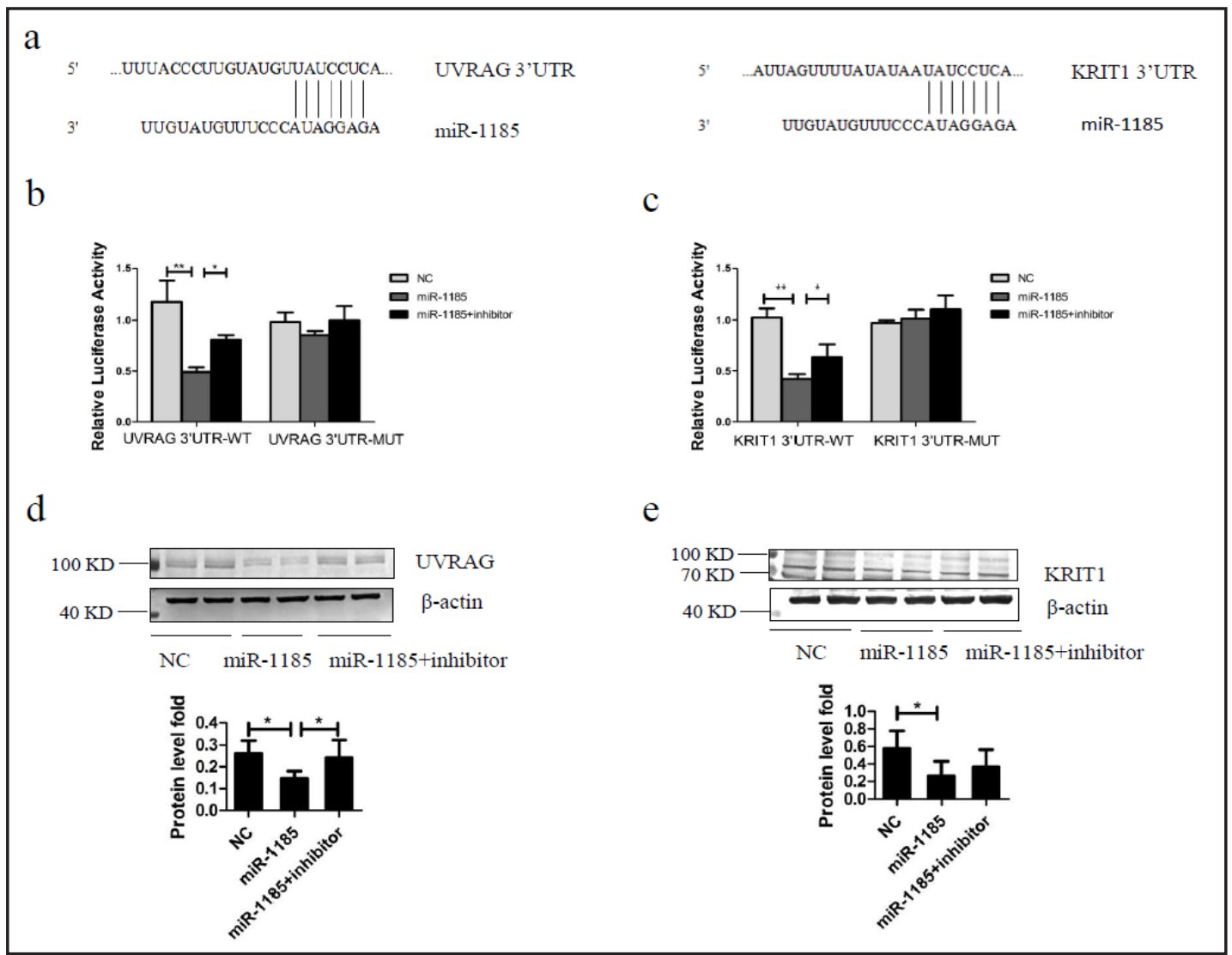

Fig. 5. Repression of UVRAG and KRIT1 expression by miR-1185. (a) Sequence alignment between miR1185 and 3' -UTR of UVRAG and KRIT1. Effects of miR-1185 on 3'-UTR of UVRAG (b) and KRIT1 (c) were determined by Luciferase activity assay. HEK-293 cells were co-transfected with constructed pMIR-UVRAG/KRIT1-3'-UTR wild-type (WT) or mutated (MUT) plasmid, miR-1185 and its inhibitor by lipofectamine 2000. After $24 \mathrm{~h}$ of transfection, luciferase activities were measured. The expression of UVRAG (d) and KRIT1 (e) were measured in pHUVEC treated with different transfection. pHUVEC were transfected with miR-1185 mimic or co-transfected with miR-1185 and its inhibitor. The scrambled sequence was used as a negative control (NC). After $48 \mathrm{~h}$ of transfection, protein expressions of UVRAG and KRIT1 were estimated by western blot. Each test was performed three times. ${ }^{*} P<0.05$ and ${ }^{* *} P<0.01$ for the indicated comparison.

in the 3'-UTR of UVRAG or KRIT1 was a very good match for the miR-1185 seed (Fig. 5a). Moreover, a luciferase assay showed that miR-1185 overexpression inhibited luciferase activity in HEK-293 cells transfected with a plasmid carrying the wild-type (WT) 3'-UTR of the UVRAG or KRIT1 gene, and co-application with miR-1185 inhibitor partly depleted the effect of miR-1185 on luciferase activity. Furthermore, the luciferase activity of the mutated (MUT) UVRAG or KRIT1 3'-UTR vector was unaffected by simultaneous transfection with miR-1185 (Fig. 5b and c). Collectively, the above data suggest that the UVRAG and KRIT1 transcripts are genuine targets of miR-1185.

To further confirm our luciferase results, similar transfection procedures were conducted in pHUVEC. The overexpression of miR-1185 decreased the UVRAG and KRIT1 protein levels compared with the NC group, and co-treatment with miR-1185 inhibitor partly attenuated the miR-1185-mediated down-regulation of UVRAG and KRIT1 expression (Fig. $5 \mathrm{~d}$ and e).

Additionally, we measured the protein expression levels of UVRAG and KRIT1 in pHUVEC, HUVSMC and THP-1 cells treated with stearic acid. Compared with the control group, the UVRAG and KRIT1 expression levels were decreased to varying degrees in cells treated with stearic acid (see supplementary material, Fig. S4a-f). 
a

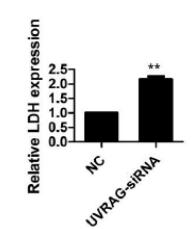

c

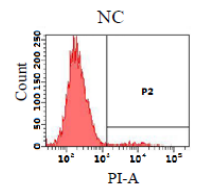

d

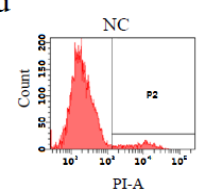

b

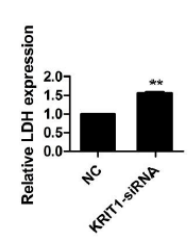

e

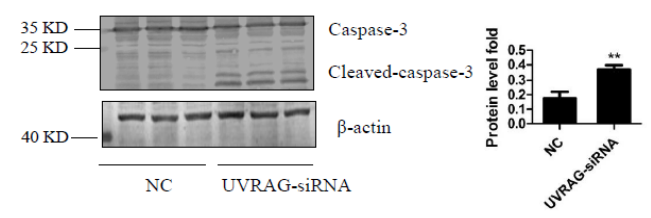

$\mathrm{f}$
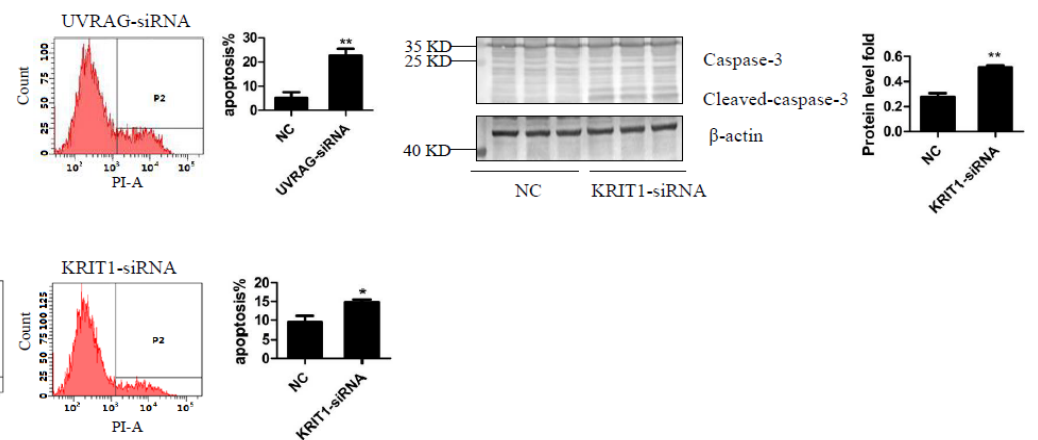

Fig. 6. Apoptotic effect of UVRAG or KRIT1 depletion by siRNA in pHUVEC. The changes in LDH release in pHUVEC transfected with UVRAG-siRNA (a) or KRIT1-siRNA (b). Percentage of apoptosis was estimated by flow cytometry with PI staining in pHUVEC treated by UVRAG-siRNA (c) or KRIT1-siRNA (d). The protein expressions of cleaved caspase-3 in pHUVEC treated by UVRAG-siRNA (e) or KRIT1-siRNA (f). The scrambled sequence was used as a negative control (NC). Each test was performed three times. $* P<0.05$ and ${ }^{* *} P$ $<0.01$ vs NC group.

\section{Depletion of UVRAG or KRIT1 resulted in pHUVEC apoptosis}

To further verify the positive effect of UVRAG and KRIT1 on endothelial cell apoptosis, pHUVEC were transfected with UVRAG-siRNA or KRIT1-siRNA, and relevant indicators of apoptosis were assessed. The transfection of UVRAG-siRNA or KRIT1-siRNA effectively reduced UVRAG or KRIT1 protein expression (see supplementary material, Fig. S5a,b), which verified the knockdown efficiency of the siRNAs targeting UVRAG and KRIT1. As shown in Fig. 6, the specific knockdown of UVRAG or KRIT1 markedly increased LDH release (Fig. 6a and b). Moreover, a flow cytometry assessment of apoptosis based on PI staining showed that the down-regulation of UVRAG or KRIT1 by siRNA significantly enhanced the percentage of apoptotic cells (Fig. 6c and d). Furthermore, a Western blot analysis demonstrated that silencing UVRAG or KRIT1 by siRNA strongly up-regulated cleaved caspase-3 protein expression (Fig. 6e and f). These results were consistent with those of the miR-1185 overexpression experiment, suggesting silencing UVRAG or KRIT1 can cause injury and apoptosis in endothelial cells.

\section{Discussion}

The present study demonstrated for the first time that miR-1185 plays a crucial role in modulating endothelial apoptosis. Specifically, the overexpression of miR-1185 significantly induced apoptosis in pHUVEC, EA.hy926 cells and HAEC. The study of the mechanism underlying this effect revealed that miR-1185 promotes endothelial cell apoptosis by suppressing the protein expression of UVRAG and KRIT1. Overall, our study provides evidence to suggest that miR-1185 may be a novel target for the prevention and treatment of atherosclerosis.

Atherosclerosis is an intricate disease of medium and large-sized arteries [27]. Importantly, the first step the pathogenesis of atherosclerosis appears to be vascular 


\section{Cellular Physiology Cell Physiol Biochem 2017;41:2171-2182 \\ \begin{tabular}{ll|l} 
DOI: 10.1159/000475571 & O 2017 The Author(s). Published by S. Karger AG, Basel \\
www.karger.com/cpb
\end{tabular} \\ Deng et al.: MiR-1185 Induces Endothelial Apoptosis}

injury [2, 28], and endothelial apoptosis plays a vital role in endothelial dysfunction and atherosclerosis lesion formation [29]. Prior to the development of atherosclerotic lesions, endothelial cell apoptosis is thought to reflect an early phase of the development of atherosclerosis, which supports the clinical concept of endothelial dysfunction preceding the formation of atherosclerotic plaques [30]. Although the mechanisms of endothelial cell apoptosis induced by multiple pro-atherosclerotic factors have been widely described [31, $32]$, the precise molecular mechanism underlying this effect requires further exploration.

Some studies have shown that the aberrant expression of miRNAs is involved in modulating endothelial apoptosis [33-35]. In this study, a microarray analysis was performed in pHUVEC treated with stearic acid, which identified miR-1185 as highly up-regulated among a panel of 48 up-regulated miRNAs. Furthermore, qPCR verified that miR-1185 was significantly increased by stearic acid and palmitic acid, which are important saturated fatty acids that are commonly considered to induce endothelial injury and apoptosis [36]. Thus, we hypothesized that miR-1185 plays an essential role in regulating endothelial cell apoptosis. Our results showed that miR-1185 promoted apoptosis in endothelial cells, including pHUVEC, EA.hy926 cells and HAEC. The apoptosis of vascular smooth muscle cells and macrophages also plays crucial roles in the development of atherosclerosis [37], but our results showed that miR-1185 did not affect apoptosis in HUVSMC and THP-1 macrophages. Therefore, miR-1185 may specifically promote the endothelial cell apoptosis.

To verify that miR-1185 directly targets genes involved in miR-1185-induced endothelial apoptosis, we predicted the targets of miR-1185 using TargetScan 6.2. Because more than two thousand genes were predicted to be potential targets of miR-1185, only genes with a good TargetScan context score that have been shown to participate in cell apoptosis were considered in this study.

UVRAG has been shown to play a critical role in modulating apoptosis in cancers in vitro and in vivo [25]. However, the mechanism underlying this effect appears to be complex. Specifically, Yin et al. $[25,38]$ reported that UVRAG, a BAX suppressor, prevents BAX-induced apoptosis by interfering with BAX activation, whereas Liang et al. [39] proved that UVRAG activates autophagy by interacting with Beclin 1, which inhibits tumourigenesis. These studies indicate that UVRAG may suppress apoptosis and activate autophagy in cancer cells. However, the ability of UVRAG to mediate endothelial cell apoptosis has not yet been verified.

The KRIT1 gene is known to be responsible for Cerebral Cavernous Malformations, which are a diffuse cerebrovascular disease characterized by abnormal dilated intracranial capillaries that are predisposed to haemorrhage. Recent studies have shown that KRIT1 is a crucial regulator of endothelial cell-cell junctions and is involved in intracellular ROS homeostasis to prevent ROS-mediated cellular dysfunction [40, 41]. In zebrafish, a loss of KRIT1 increases endothelial apoptosis during microvascular angiogenesis, and KRIT1 deficiency promotes apoptosis in HUVEC by reducing activation of the integrin-linked kinase survival signalling pathway [26, 42], suggesting that KRIT1 plays a critical role in the endothelial apoptosis.

In this study, the overexpression of miR-1185 strongly repressed the protein expression of UVRAG and KRIT1, and this process was followed by endothelial cell apoptosis, which was similar to that observed when UVRAG or KRIT1 was inhibited with specific siRNA. These findings also corroborated those of other studies regarding the roles of UVRAG [25] and KRIT1 [42] in apoptosis, indicating that UVRAG and KRIT1, which are direct targets of miR1185 , are involved in the mechanism of miR-1185-induced endothelial apoptosis.

In addition, stearic acid increased miR-1185 expression in endothelial cells but decreased miR-1185 expression in HUVSMC and THP-1 cells. These results reveal that stearic acid-induced miR-1185 regulation may depend on the cell type. Unexpectedly, stearic acid reduced miR-1185 levels in HUVSMC and THP-1 cells, which was accompanied by decreases in UVRAG and KRIT1. miRNAs are known to target many mRNAs, and one mRNA can be regulated by multiple miRNAs. Therefore, the reductions in UVRAG and KRIT1 expression may be mediated by other miRNAs or unknown factors in HUVSMC and THP-1 cells treated with stearic acid. 
To our knowledge, this report is the first to suggest that miR-1185 has pro-apoptosis effects on endothelial cells by directly targeting UVRAG and KRIT1. Specifically, our study identifies a novel mechanism related to endothelial apoptosis: increases in miR-1185 suppress the protein expression levels of UVRAG and KRIT1, which induces endothelial apoptosis, and may promote the development of atherosclerosis. These findings contribute to a better understanding of endothelial apoptosis and atherosclerotic pathogenesis and provide a new potential insight into therapeutic approaches in atherosclerosis. However, the effect of other factors on miR-1185, such as genes and other stimuli, requires further investigation.

\section{Limitation}

This study aimed to illuminate the effects of miR-1185 on endothelial apoptosis during the development of atherosclerosis and the mechanism underlying this effect. To clearly identify the influence of miR-1185 on endothelial cells and related mechanisms, different endothelial cell types, i.e., pHUVEC, EA.hy926 cells and HAEC, were used in this study. In addition, the pro-apoptotic effect of miR-1185 was also explored in HUVSMC and THP-1 macrophages. The application of multiple cells can help to better to reflect the impacts of miR-1185 on vascular injury and the pathogenesis of atherosclerosis. However, our study was also subject to limitations. First, since species-specific miR-1185 was not expressed in mice and rats, we only proved the pro-apoptotic effects and mechanisms of miR-1185 in vitro. Second, the pathological and physiological process regulated by miRNAs is complex. Thousands of potential target genes of miR-1185 were predicted by database, and the involvement of other target genes in the process requires further study. Finally, because we did not recruit a sufficient number of patients with atherosclerosis, the precise effect of miR1185 on atherosclerosis remains to be further validated in individuals.

\section{Acknowledgements}

This research was supported by grants from the National Natural Science Foundation of China (No. 81673153 and 81472980) and the Province in Heilongjiang Outstanding Youth Science Fund (JC201410).

\section{Disclosure Statement}

The authors declare no conflict of interests.

\section{References}

1 Lovren F, Pan Y, Quan A, Singh KK, Shukla PC, Gupta N, Steer BM, Ingram AJ, Gupta M, Al-Omran M: MicroRNA-145 targeted therapy reduces atherosclerosis. Circulation 2012;126:81-90.

-2 Epstein FH, Ross R: Atherosclerosis-an inflammatory disease. New Engl J Med 1999;340:115-126.

-3 Dimmeler S, Hermann C, Zeiher AM: Apoptosis of endothelial cells. Contribution to the pathophysiology of atherosclerosis? Eur Cytokine Netw 1998;9:697-698.

4 Lüscher TF, Barton M: Biology of the endothelium. Clin Cardiol 1997;20:II-3-10.

5 Xu Q: The impact of progenitor cells in atherosclerosis. Nat Clin Pract Card 2006;3:94-101.

6 Pober JS, Min W, Bradley JR: Mechanisms of endothelial dysfunction, injury, and death. Annu Rev Pathol 2009;4:71-95. 


\section{Cellular Physiology Cell Physiol Biochem 2017;41:2171-2182

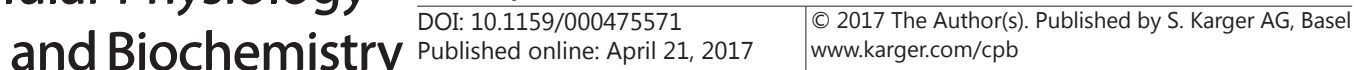 \\ Deng et al.: MiR-1185 Induces Endothelial Apoptosis}

7 Berliner J, Territo M, Sevanian A, Ramin S, Kim JA, Bamshad B, Esterson M, Fogelman AM: Minimally modified low density lipoprotein stimulates monocyte endothelial interactions. J Clin Invest 1990;85:12601266.

8 Stadtman ER: Importance of individuality in oxidative stress and aging. Free Radic Biol Med 2002;33:597604.

-9 Bartel DP: MicroRNAs: genomics, biogenesis, mechanism, and function. Cell 2004;116:281-297.

10 Wronska A, Kurkowska-Jastrzebska I, Santulli G: Application of microRNAs in diagnosis and treatment of cardiovascular disease. Acta Physiol (Oxf) 2015;213:60-83.

-11 Santulli G: microRNAs and Endothelial (Dys) Function. J Cell Physiol 2016;231:1638-1644.

12 Xu Y, Zhu W, Wang Z, Yuan W, Sun Y, Liu H, Du Z: Combinatorial MicroRNAs Suppress Hypoxia-Induced Cardiomyocytes Apoptosis. Cell Physiol Biochem 2015;37:921-932.

13 Santulli G: microRNAs Distinctively Regulate Vascular Smooth Muscle and Endothelial Cells: Functional Implications in Angiogenesis, Atherosclerosis, and In-Stent Restenosis. Adv Exp Med Biol 2015;887:53-77.

14 Novák J, Olejníčková V, Tkáčová N, Santulli G: Mechanistic Role of MicroRNAs in Coupling Lipid Metabolism and Atherosclerosis. Adv Exp Med Biol 2015;887:79-100.

15 Santulli G, Wronska A, Uryu K, Diacovo TG, Gao M, Marx SO, Kitajewski J, Chilton JM, Akat KM, Tuschl T: A selective microRNA-based strategy inhibits restenosis while preserving endothelial function. J Clin Invest 2014;124:4102-4114.

16 Magenta A, Cencioni C, Fasanaro P, Zaccagnini G, Greco S, Sarra-Ferraris G, Antonini A, Martelli F, Capogrossi MC: miR-200c is upregulated by oxidative stress and induces endothelial cell apoptosis and senescence via ZEB1 inhibition. Cell Death Differ 2011;18:1628-1639.

17 Zhang T, Tian F, Wang J, Jing J, Zhou SS, Chen YD: Atherosclerosis-Associated Endothelial Cell Apoptosis by MiR-429-Mediated Down Regulation of Bcl-2. Cell Physiol Biochem 2015;37:1421-1430.

- 18 Ye M, Li D, Yang J, Xie J, Yu F, Ma Y, Zhu X, Zhao J, Lv Z: MicroRNA-130a Targets MAP3K12 to Modulate Diabetic Endothelial Progenitor Cell Function. Cell Physiol Biochem 2015;36:712-726.

19 Zhou Z, You Z: Mesenchymal Stem Cells Alleviate LPS-Induced Acute Lung Injury in Mice by MiR-142a-5pControlled Pulmonary Endothelial Cell Autophagy. Cell Physiol Biochem 2016;38:258-266.

20 Artwohl M, Lindenmair A, Sexl V, Maier C, Rainer G, Freudenthaler A, Huttary N, Wolzt M, Nowotny P, Luger A, Baumgartner-Parzer SM: Different mechanisms of saturated versus polyunsaturated FFA-induced apoptosis in human endothelial cells. J Lipid Res 2008;49:2627-2640.

21 Freedman JE, Ercan B, Morin KM, Liu C-T, Tamer L, Ayaz L, Kanadasi M, Cicek D, Seyhan AI, Akilli RE: The distribution of circulating microRNA and their relation to coronary disease. F1000Res 2012;1:50.

-22 Lu H, Hao L, Li S, Lin S, Lin L, Chen Y, Cui H, Zi T, Xia C, Na L: Elevated circulating stearic acid leads to a major lipotoxic effect on mouse pancreatic beta cells in hyperlipidaemia via a miR-34a-5p-mediated PERK/ p53-dependent pathway. Diabetologia 2016;59:1247-1257.

-23 Artwohl M, Roden M, Waldhäusl W, Freudenthaler A, Baumgartner-Parzer SM: Free fatty acids trigger apoptosis and inhibit cell cycle progression in human vascular endothelial cells. FASEB J 2004;18:146-148.

-24 Chai W, Liu Z: p38 mitogen-activated protein kinase mediates palmitate-induced apoptosis but not inhibitor of nuclear factor-kappaB degradation in human coronary artery endothelial cells. Endocrinology 2007;148:1622-1628.

25 Yin X, Cao L, Kang R, Yang M, Wang Z, Peng Y, Tan Y, Liu L, Xie M, Zhao Y, Livesey KM, Tang D: UV irradiation resistance-associated gene suppresses apoptosis by interfering with BAX activation. EMBO Rep 2011;12:727-734.

26 Liu H, Rigamonti D, Badr A, Zhang J: Ccm1 assures microvascular integrity during angiogenesis. Transl Stroke Res 2010;1:146-153.

27 Ding Z, Liu S, Wang X, Khaidakov M, Dai Y, Mehta JL: Oxidant stress in mitochondrial DNA damage, autophagy and inflammation in atherosclerosis. Sci Rep 2013;3:1077.

28 Ross R: The pathogenesis of atherosclerosis: a perspective for the 1990s. Nature 1993;362:801-809.

29 Alvarez RJ, Gips SJ, Moldovan N, Wilhide CC, Milliken EE, Hoang AT, Hruban RH, Silverman HS, Dang CV, Goldschmidt-Clermont PJ: 17 $\beta$-Estradiol inhibits apoptosis of endothelial cells. Biochem Biophys Res Commun 1997;237:372-381.

-30 Schächinger V, Britten MB, Zeiher AM: Prognostic impact of coronary vasodilator dysfunction on adverse long-term outcome of coronary heart disease. Circulation 2000;101:1899-1906. 


\section{Cellular Physiology Cell Physiol Biochem 2017;41:2171-2182

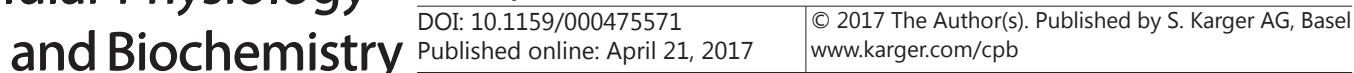 \\ Deng et al.: MiR-1185 Induces Endothelial Apoptosis}

-31 Baumgartnerparzer SM, Wagner L, Pettermann M, Grillari J, Gessl A, Waldhäusl W: High-glucose--triggered apoptosis in cultured endothelial cells. Diabetes 1995;44:1323-1327.

-32 Dimmeler S, Haendeler J, Galle J, Zeiher AM: Oxidized low-density lipoprotein induces apoptosis of human endothelial cells by activation of CPP32-like proteases. A mechanistic clue to the 'response to injury' hypothesis. Circulation 1997;95:1760-1763.

-33 Weber M, Baker MB, Moore JP, Searles CD: MiR-21 is induced in endothelial cells by shear stress and modulates apoptosis and eNOS activity. Biochem Biophys Res Commun 2010;393:643-648.

34 Qin B, Xiao B, Liang D, Li Y, Jiang T, Yang H: MicroRNA let-7c inhibits Bcl-xl expression and regulates oxLDL-induced endothelial apoptosis. BMB Rep 2012;45:464-469.

35 Bao H, Gao F, Xie G, Liu Z: Angiotensin-converting enzyme 2 inhibits apoptosis of pulmonary endothelial cells during acute lung injury through suppressing mir-4262. Cell Physiol Biochem 2015;37:759-767.

36 Harvey KA, Walker CL, Pavlina TM, Xu Z, Zaloga GP, Siddiqui RA: Long-chain saturated fatty acids induce pro-inflammatory responses and impact endothelial cell growth. Clin Nutr 2010;29:492-500.

-37 Schrijvers DM, De Meyer GR, Kockx MM, Herman AG, Martinet W: Phagocytosis of Apoptotic Cells by Macrophages Is Impaired in Atherosclerosis. Arterioscler Thromb Vasc Biol 2005;25:1256-1261.

-38 Yin X, Cao L, Peng Y, Tan Y, Xie M, Kang R, Livesey KM, Tang D: A critical role for UVRAG in apoptosis. Autophagy 2011;7:1242-1244.

-39 Liang C, Feng P, Ku B, Dotan I, Canaani D, Oh BH, Jung JU: Autophagic and tumour suppressor activity of a novel Beclin1-binding protein UVRAG. Nat Cell Biol 2006;8:688-699.

40 Glading A, Han J, Stockton RA, Ginsberg MH: KRIT-1/CCM1 is a Rap1 effector that regulates endothelial cell cell junctions. J Cell Biol 2007;179:247-254.

41 Goitre L, Balzac F, Degani S, Degan P, Marchi S, Pinton P, Retta SF: KRIT1 regulates the homeostasis of intracellular reactive oxygen species. PLoS One 2010;5:e11786.

-42 Hogan BM, Bussmann J, Wolburg H, Schultemerker S: ccm1 cell autonomously regulates endothelial cellular morphogenesis and vascular tubulogenesis in zebrafish. Hum Mol Genet 2008;17:2424-2432. 